\title{
Carcinogenic Intervention
}

National Cancer Institute

\section{Source}

National Cancer Institute. Carcinogenic Intervention. NCI Thesaurus. Code C41078.

Treatment or procedure that the animal model was exposed to in order to initiate or support the development of neoplastic lesions. 